\title{
Perioperative outcomes: easier to predict but harder to change
}

\author{
Karim S. Ladha, MD, MSc • Duminda N. Wijeysundera, MD, PhD
}

Received: 10 May 2019/Revised: 10 May 2019/Accepted: 17 June 2019/Published online: 25 June 2019

(C) Canadian Anesthesiologists' Society 2019

Whether choosing to walk down a street or jump out of an airplane, nearly every decision that we take in life confers some associated degree of risk. Given the frequency with which we must weigh risk versus benefit in our daily lives, we tend to make these judgements rapidly and implicitly without any formal mathematical computation. This intuitive approach is typically sufficient because many of the risks we assume during our daily lives are miniscule. In contrast, the perioperative period can pose risks with potentially life-altering consequences, thereby making surgery-related decisions much more consequential.

Despite the importance of perioperative decisionmaking, both anecdotal and clinical evidence suggest that physicians largely rely on intuition and implicit processing to risk-stratify patients with respect to potential postoperative adverse events. ${ }^{1}$ Nevertheless, clinical judgement is often inaccurate and inconsistent when evaluating potential clinical consequences. ${ }^{2-4}$ The absence of routine formal risk stratification is not for lack of scores or tools. For example, considering cardiac surgery alone, there are at least 19 risk models that estimate the probability of mortality for a particular patient. ${ }^{5}$

K. S. Ladha, MD, MSc · D. N. Wijeysundera, MD, PhD (ه) Department of Anesthesia, St. Michael's Hospital, 30 Bond Street, Toronto, ON M5B 1W8, Canada

e-mail: d.wijeysundera@utoronto.ca

Li Ka Shing Knowledge Institute of St. Michael's Hospital, Toronto, ON, Canada

Department of Anesthesia, University of Toronto, Toronto, ON, Canada

Institute of Health Policy Management and Evaluation, University of Toronto, Toronto, ON, Canada
In this era of evidence-based medicine, as clinicians become increasingly familiar with formal risk scores and their incorporation into perioperative risk estimation, a key point to address is the incremental benefit that is gained by calculating risk. In other words, does estimating the probability of an event change a patient's perioperative course? The extensive literature concerned with risk estimation is rooted in the assumption that more accurate measurement of probability will translate into improved care and outcome. The theoretical improvements in perioperative care derived from formal risk prediction are much-needed advancements that are likely to garner wide support. For example, shifting scarce resources to patients who are most likely to benefit, or improving shared decision-making, are laudable goals that are often cited as likely benefits of risk stratification. Nevertheless, proving that measuring risk is beneficial in the real world has been substantially more difficult than developing the models themselves.

In this issue of the Journal, Harris et al. present a scoping review summarizing published evidence pertaining to the ability of risk prediction tools to affect outcomes. ${ }^{6}$ They systematically sought out analyses that examined the impact of conveying risk probability to both patients and health care providers. Despite extensive searching, only seven studies were identified. Of these seven, only three were published as full manuscripts with the remainder never progressing forward from abstract form. Often, no statistical tests were performed to quantify the risk impact of the intervention, and only two studies used externally validated models.

While the authors conducted a high-quality scoping review, the paucity of evidence limits the conclusions that can be made. Nonetheless, their review is an important contribution to the literature since it highlights a serious 
knowledge deficit within perioperative medicine and provides guidance on how to direct future research. We must move beyond developing risk prediction models with ever increasing accuracy and complexity to understanding their potential impact on clinical practice. While adequate discrimination, calibration, and validation are prerequisites for any useful model, these metrics are not sufficient to confer utility. Determining that a patient's risk of 30-day mortality is $30 \%$ is arguably of questionable utility unless it triggers an action. The current state of evidence leaves us wondering what this estimate should prompt: a conversation, a consult, or even a cancellation?

To better inform clinical practice, future studies need to address several key shortcomings within the current paradigm of perioperative risk prediction. First, risk prediction should not be considered in isolation. Rather, risk estimates must be bundled with decision-making models or targeted interventions. The efficacy of this "bundled" approach can then be rigorously tested using randomized-controlled trials with appropriate outcomes. ${ }^{7}$ Second, where appropriate, risk estimates should be considered as a dynamic rather than a static metric. In other words, adoption of an iterative approach to calculating probabilities where risk estimates are updated as more information becomes available. Bayesian statistical methods are particularly well-suited to integrate new data with prior risk estimates, but these methods remain uncommon in clinical research and decisionmaking.

Third, additional consideration needs to be applied to the patient-centric outcomes of interest. Certainly, death and stroke are adverse events that all patients will want to avoid; however, there are other crucial concerns such as maintaining an acceptable quality of life and the ability to live independently at home. These outcomes are often the "downstream" result of perioperative clinical decisions, but postoperative function may decrease even in the absence of a complication. While these are outcomes that are clearly important to patients, they are not adequately considered by most existing risk prediction tools.

Finally, in their manuscripts title, Harris et al. use the phrase "Personalized Perioperative Medicine". The term conjures up thoughts of complex genetic analysis and sophisticated disease modelling enabled by advances in biotechnology and data processing. These technological advances undoubtedly offer exciting possibilities to revolutionize and better individualize perioperative care. Yet, personalized medicine must place equal weight on appropriate communication skills amongst healthcare providers. The ability to communicate risk (and its implications) is just as important as the ability to calculate it. Considering the familiar scenario of an anxious patient weighing potentially life-altering choices about upcoming surgery, we must understand the patients' beliefs and values to deliver care that is congruent with their priorities. This is rarely achieved within the current model of healthcare; ideally the patient should meet with a range of perioperative healthcare providers (e.g., physicians, nurses, physiotherapists) who can provide timely and appropriate information that can help the patient to make better-informed decisions regarding the planned surgical intervention. It is lamentable that anesthesiologists are currently in the position of having to address perioperative risk, sometimes just minutes before surgery.

With improving surgical and anesthesia techniques and evolving surgical care pathways, we now consider older, frailer patients for surgery who previously would have been regarded as unfit. Risk stratification offers the possibility to provide patient-centred care, improved outcomes, and increased efficiency. The scoping review by Harris et al. demonstrates that more high-quality research is needed if accurate risk prediction is to fulfil its theoretical promise. Equally important is the need to change risk prediction from an analytical issue (i.e., reporting the most accurate risk estimate possible) to an integral component of the preoperative evaluation process. It is crucial that risk prediction delivers value-based individualized care in the context of a multidisciplinary environment, where patients have sufficient time and information to fully consider options at this crucial juncture in their medical journey.

Que nous décidions d'emprunter une rue plutôt qu'une autre ou de sauter d'un avion, presque toutes les décisions que nous prenons dans la vie comportent un certain degré de risque. Étant donné la fréquence à laquelle nous devons évaluer les risques versus les avantages dans notre vie quotidienne, nous avons tendance à prendre ces décisions rapidement et implicitement, sans calcul mathématique formel. En règle générale, cette approche intuitive est bien suffisante, car bon nombre des risques que nous prenons chaque jour sont minimes. En revanche, la période périopératoire peut poser des risques impliquant des conséquences potentiellement catastrophiques: les décisions liées à la chirurgie ont donc une implication bien plus importante.

\section{Pronostics périopératoires : plus faciles à prédire, plus difficiles à modifier}

Malgré l'importance de la prise de décision périopératoire, les données probantes anecdotiques et cliniques suggèrent toutes deux que les médecins 
s'appuient en grande partie sur leur intuition et sur des processus implicites pour stratifier les patients en fonction de leur risque en ce qui touche aux événements indésirables postopératoires potentiels. ${ }^{1}$ Toutefois, le jugement clinique est bien souvent imprécis et incohérent lorsqu'on évalue les conséquences cliniques potentielles. ${ }^{2-4}$ L'absence de stratification formelle et systématique des risques n'est pas due au manque de scores ou d'outils. Par exemple, si l'on considère la chirurgie cardiaque, il existe au moins 19 modèles de risque pour estimer la probabilité de mortalité d'un patient en particulier. ${ }^{5}$

Dans notre ère de médecine fondée sur des données probantes, alors que les cliniciens sont de plus en plus au fait des scores de risque formels et de leur intégration dans l'estimation des risques périopératoires, un élément clé à aborder est l'avantage que l'on acquiert en calculant le risque. Autrement dit, est-ce que le parcours périopératoire d'un patient sera modifié si l'on estime la probabilité d'un événement? La vaste littérature qui s'intéresse à l'estimation du risque part du principe qu'une mesure plus précise de la probabilité se traduira en des soins et des pronostics améliorés. Les améliorations théoriques en soins périopératoires dérivées d'une prédiction formelle du risque sont des améliorations plus que bienvenues qui seront probablement soutenues par tous. Par exemple, le fait d'offrir des ressources rares aux patients qui en bénéficieraient probablement le plus, ou l'amélioration de la prise de décision partagée - voilà deux objectifs louables souvent cités comme avantages probables d'une stratification des risques. Cependant, il s'est avéré bien plus difficile de prouver qu'il est bénéfique de mesurer le risque dans la vraie vie que de mettre au point les modèles pour le faire.

Dans ce numéro du Journal, Harris et coll. présentent un compte-rendu résumant les données probantes publiées concernant la capacité des outils de prédiction de risque à affecter les pronostics. ${ }^{6}$ Les auteurs ont recherché de façon systématique les analyses examinant l'impact de la communication de la probabilité de risque aux patients et aux professionnels de la santé. Malgré des recherches exhaustives, seules sept études ont pu être identifiées. Parmi ces sept études, seules trois ont été publiées en tant que manuscrits complets, alors que les autres n'ont pas dépassé le stade de résumé. Bien souvent, aucun test statistique n'a été réalisé afin de quantifier l'impact de risque de l'intervention, et seulement deux études ont utilisé des modèles validés.

Bien que les auteurs aient réalisé une étude de portée de haute qualité, le peu de données probantes limite les conclusions qui peuvent en être tirées. Leur compte rendu demeure néanmoins une contribution importante à la littérature, étant donné qu'il met en lumière un important déficit de connaissances au sein de la médecine périopératoire et offre des pistes pour les recherches futures. Nous devons aller plus loin que la simple élaboration de modèles de prédiction du risque avec une précision et une complexité toujours grandissantes, et nous diriger vers la compréhension de leur impact potentiel sur la pratique clinique. Bien qu'une discrimination, un calibrage et une validation adéquats sont des prérequis de tout modèle bien construit, ces mesures ne suffisent pas à lui conférer une utilité véritable. Quelle est l'utilité réelle de déterminer que le risque de mortalité à 30 jours d'un patient est de $30 \%$ si cela ne déclenche pas une réaction? L'état actuel des données probantes soulève la question de savoir ce qu'une telle estimation devrait déclencher : une conversation, une consultation, voire une annulation?

Afin de mieux aider la pratique clinique, les études futures doivent pallier plusieurs écueils clés qui se retrouvent au sein du paradigme actuel de prédiction du risque périopératoire. Tout d'abord, la prédiction du risque ne devrait pas être examinée de manière isolée. Plutôt, les estimations de risque doivent être liées à des modèles de prise de décision ou à des interventions ciblées. L'efficacité de cette approche 'regroupée' peut ensuite être testée de façon rigoureuse dans des études randomisées contrôlées possédant des critères d'évaluation adaptés. ${ }^{7}$ Deuxièmement, lorsque possible, les estimations de risque devraient être envisagées en tant que mesures dynamiques plutôt que statiques. Autrement dit, il faudrait adopter une approche itérative du calcul des probabilités, dans laquelle les estimations de risque sont mises à jour au fur et à mesure que de nouvelles informations sont disponibles. Les méthodes statistiques bayésiennes sont particulièrement utiles pour intégrer de nouvelles données à des estimations antérieures de risque, mais ces méthodes demeurent encore peu répandues dans la recherche clinique et la prise de décision.

Troisièmement, il faut porter une attention supplémentaire aux critères d'évaluation centrés sur le patient. Il ne fait aucun doute que la mort et les accidents vasculaires cérébraux constituent des événements indésirables que tout patient voudra éviter; toutefois, il existe d'autres inquiétudes cruciales telles que le maintien d'une qualité de vie acceptable et la capacité de vivre de façon autonome à la maison. Ces résultats sont trop souvent la résultante a posteriori de décisions cliniques périopératoires, mais la fonctionnalité postopératoire pourrait baisser et ce, même en l'absence de complications. Bien que ces pronostics soient clairement 
importants pour les patients, ils ne sont pas adéquatement mesurés par la plupart des outils de prédiction de risque existants.

Enfin, dans le titre de leur manuscrit, Harris et coll. utilisent l'expression «Médecine périopératoire personnalisée ». Cette expression évoque des images d'analyse génétique complexe et de modèles de morbidité sophistiqués rendus possibles grâce aux progrès de la biotechnologie et du traitement de données. Ces progrès technologiques offrent incontestablement des possibilités excitantes de révolutionner et de mieux personnaliser les soins périopératoires. La médecine personnalisée doit toutefois accorder autant d'importance à des habiletés de communication adaptées parmi les fournisseurs de soins de santé. La capacité à communiquer un risque (et ses implications) est tout aussi importante que la capacité à le calculer. Si l'on pense au scénario bien connu d'un patient anxieux examinant les choix potentiellement fatidiques d'une chirurgie future, nous devons comprendre les valeurs et les convictions dudit patient pour pouvoir lui offrir des soins qui s'alignent avec ses priorités. C'est rarement le cas dans le modèle de soins de santé actuel; idéalement, le patient devrait rencontrer plusieurs fournisseurs de soins de santé périopératoires (par ex. médecins, infirmières, physiothérapeutes) qui pourront lui donner des informations adéquates et opportunes qui peuvent l'aider à prendre des décisions mieux informées quant à l'intervention chirurgicale prévue. Il est déplorable qu'il arrive que les anesthésiologistes doivent aborder le risque périopératoire parfois quelques minutes seulement avant la chirurgie.

Grâce aux progrès des techniques chirurgicales et anesthésiques et aux nouveaux protocoles de soins chirurgicaux, nous envisageons désormais d'opérer des patients plus âgés et plus fragiles qu'auparavant. La stratification des risques nous donne l'occasion d'offrir des soins centrés sur le patient, de meilleurs pronostics, et une efficacité accrue. Le compte-rendu de portée de Harris et coll. démontre que d'autres recherches de qualité élevée sont nécessaires si l'on souhaite qu'une prédiction précise du risque remplisse ses promesses théoriques. Il est tout aussi important de modifier le statut de la prédiction du risque, soit d'un problème analytique (c'est-à-dire, la communication d'une estimation la plus précise possible $\mathrm{du}$ risque) à une composante intégrale du processus d'évaluation préopératoire. Il est essentiel que la prédiction du risque offre des soins personnalisés fondés sur la valeur dans le contexte d'un environnement multidisciplinaire, dans lequel les patients ont suffisamment de temps et d'informations pour envisager toutes les options à ce point tournant de leur évolution médicale.

Conflicts of interest None declared.

Editorial responsibility This submission was handled by Dr. Steven Backman, Associate Editor, Canadian Journal of Anesthesia.

Funding Dr. Wijeysundera is supported in part by a New Investigator Award from the Canadian Institutes of Health Research, an Excellence in Research Award from the Department of Anesthesia at the University of Toronto, and the Endowed Chair in Translational Anesthesiology Research at St. Michael's Hospital and University of Toronto. Dr. Ladha is supported in part by a Merit Award from the Department of Anesthesia at the University of Toronto.

\section{Conflit d'intérêt Aucun.}

Responsabilité éditoriale Cet article a été traité par Dr Hilary P. Grocott, rédacteur en chef, Journal canadien d'anesthésie.

Financement Dr Wijeysundera est soutenu en partie par une Bourse salariale de nouveau chercheur des Instituts de recherche en santé du Canada, une Prime de mérite du département d'anesthésie de l'Université de Toronto, et de la Chaire dotée en recherche translationnelle en anesthésiologie à l'Hôpital St. Michael's et à l'Université de Toronto. Dr Ladha est soutenu en partie par une Prime de mérite du département d'anesthésie de l'Université de Toronto.

\section{References}

1. Devereaux PJ, Ghali WA, Gibson NE, et al. Physician estimates of perioperative cardiac risk in patients undergoing noncardiac surgery. Arch Intern Med 1999; 159: 713-7.

2. Liao L, Mark DB. Clinical prediction models: are we building better mousetraps? J Am Coll Cardiol 2003; 42: 851-3.

3. Hoffmann TC, Del Mar C. Clinicians' expectations of the benefits and harms of treatments, screening, and tests: a systematic review. JAMA Intern Med 2017; 177: 407-19.

4. Wijeysundera DN, Pearse RM, Shulman MA, et al. Assessment of functional capacity before major non-cardiac surgery: an international, prospective cohort study. Lancet 2018; 391: 2631-40.

5. Prins $C$, de Villiers Jonker I, Botes L, Smit FE. Cardiac surgery risk-stratification models. Cardiovasc J Afr 2012; 23: 160-4.

6. Harris EP, MacDonald DB, Boland L, Boet S, Lalu MM, McIsaac $D$. Personalized perioperative medicine: a scoping review of personalized assessment and communication of risk before surgery. Can J Anesth 2019; 66. https://doi.org/10.1007/s12630019-01432-6

7. Kappen TH, Vergouwe Y, van Wolfswinkel L, Kalkman CJ, Moons $K G$, Van Klei WA. Impact of adding therapeutic recommendations to risk assessments from a prediction model for postoperative nausea and vomiting. Br J Anaesth 2015; 114: 252-60.

Publisher's Note Springer Nature remains neutral with regard to jurisdictional claims in published maps and institutional affiliations. 